\title{
IoT Based Smart Lighting - Intelligent and Weather Adaptive Lighting in Street Lights for Efficient Use of Power
}

\author{
Nirmalrani V, Saravanan P, Sowmiya G, Peruma C
}

\begin{abstract}
This paper proposes tremendousness productive of canny street lighting structure using irrelevant exertion micro controller based Arduino. The central objective is to structure imperativeness capable sharp streetlight for significance confirmation in existing streetlights of fundamental zone, urban zone and just for clever urban zones. The structure contains $L E D$ luminaire, LED driver, PV board, charge controller light sensor, headway sensor, Arduino. The gifted streetlight is controlled subject to traffic on road and day/evening time. The structure is adjusted to ordinarily butcher in the midst of the essential stores of light and fundamentally work in the midst of the night and overpowering down-pouring or stunning air. All around we see that street lights are remain traded $\mathrm{ON}$ in the midst of day time, this is total of misuses of power while India is confronting nonattendance of intensity. Another issue is the standard street light for instance Sodium vapor, Metal halide, Incandescent, Fluorescent lights uses more power when risen up out of new pushed Led Lights. Streetlights can be worked free of cost by using changed controlled, self-animated, productive sun filled LED street light. The IOT gives the solid seeing of the street lights and the hugeness use. If the battery is totally charged the imperativeness passed on from the sun fueled is passed to the standard cross portion.
\end{abstract}

Index Terms: Alarm Catch, Component, Microcontroller, Road Light, Sensor.

\section{INTRODUCTION}

This paper proposes mass beneficial of competent street lighting structure using irrelevant exertion microcontroller based Arduino. The central objective is to structure essentialness gifted sharp streetlight for significance assertion in existing streetlights of primary zone, urban zone and just for savvy urban zones [12]. The structure contains LED luminaire, LED driver, PV board, charge controller light sensor, development sensor, Arduino. The dexterous streetlight is controlled subject to traffic on road and day/evening time. The structure is changed to generally butcher in the midst of the essential stores of light and fundamentally work in the midst of the night and overpowering down-pouring or amazing air [2]. By and large we see that street lights are stay traded $\mathrm{ON}$ in the midst of day time, this is total of maltreatment of power while India is

Revised Version Manuscript Received on 10 September, 2019.

Nirmalrani V, School of Computing, Sathyabama Institute of Science and Technology, Chennai, Tamilnadu, India.

Saravanan P, School of Computing, Sathyabama Institute of Science and Technology, Chennai, Tamilnadu, India.

Sowmiya G, Department of IT, Sathyabama Institute of Science and Technology, Chennai, Tamilnadu, India.

Peruma C, Department of IT, Sathyabama Institute of Science and Technology, Chennai, Tamilnadu, India. confronting nonattendance of power. Another issue is the standard street light for instance Sodium vapor, Metal halide, Incandescent, Fluorescent lights uses more power when climbed out of new pushed Led Lights. Streetlights can be worked free of cost by using changed controlled, self-vivified, valuable sun filled LED street light [12]. The IoT gives the solid seeing of the street lights and the critical use. If the battery is totally charged the essentialness passed on from the sun controlled is passed to the standard cross piece. A full scale lighting control structure contains two passing on modules: sunshine control module and phony lighting control module. The clarifications behind these two modules are phenomenal. Sunshine control module is on an uncommonly central measurement utilized for reducing noteworthiness costs while counterfeit lighting control module is basic for giving a sensibly content with workplace. Robotizing and adjusting the lighting control structure with a definitive spotlight on that uniform and stable lighting is kept up at included zones while centrality utilizes are kept as low as conceivable breezes up being a troublesome issue [1].

\section{RELATED WORK}

A. Designing an Adaptive Lighting Control System for Smart Buildings and Homes, Yuan Wang, 2015

Lighting control in cautious structures and homes can be robotized by having PC controlled lights and blinds close-by lighting up sensors that are appropriated in the building Notwithstanding, programming a tremendous building light switches and apparently crippled settings can be unpropitious and extraordinary. We present a system that algorithmically sets up the control structure that can robotize any working without custom programming. This is cleaned by making the structure self-changing and self-learning. This paper depicted how the issue is NP hard regardless can be settled by heuristics. The consequent structure controls blinds to ensure despite lighting what's more adds counterfeit illumination to ensure light breaker remains engaging constantly of the day, adjusting for condition and seasons. Without daylight, the system resorts to fake lighting. Our methods fill in as nonexclusive control computations and are not prearranged for a particular spot. The likelihood, adaptively and adaptability features of the system have been grasped through various confirmed and imitated tests [1]. 
B. IoT based smart and adaptive lighting in street lights, S.Gurupriya, 2017

The structure is on an exceptionally fundamental dimension utilized for sharp and air adaptable lighting in road lights. The endeavor is finished with sharp inserted structure that controls the road light dependent on watching validation of daylight. Amidst the night the road light gets consistently OFF. The ON/OFF can be gotten to wherever at whatever point through web. A camera is set over the road light to seek after the activities performed out on the town where the records are demanded in a server. What's more, a free for all catch is put on the post, if there should rise an occasion of any crisis or risk, the individual in danger can smash this catch which raises a caution at the adjacent police base camp. At whatever point the free for all catch is squashed, the story around then recorded by the camera is sent unequivocally to the cloud account. The bit of the record is given to the specific police focal station by which they can see the occasion's spot. Each zone's road lights are associated with the specific region's police home office and every one of them have a cloud available record. The manual endeavor utilizing GSM advancement is totally disposed of. Accordingly the structure is for the most part proposed to guarantee security and to imagine hugeness wastage.

\section{Smart Lighting for Smart Cities, Vijay Barve, 2017}

While the abundance of a mechanical gathering gets picked by the redesign yet we for the most part need to channel for progress of usage by restricting human impedance in the midst of upgrade and control. The paper gives information in to control of lighting structure unequivocally with least human check. Sharp lighting structure unites the Main server, neighborhood controllers and LED lighting establishments. For street lighting structures, the correspondence between Main server and close controllers is through GSM make while that between neighborhood controllers and lighting mechanical social affairs is in two clear courses via through power cabling framework or through RF correspondence. For indoor lighting structures for the instance of essential server inside the premises, the correspondence between the areas of the mindful lighting system is either hard wired or through RF correspondence. Varying sorts of sensors are given like photo sensor, enhancement sensor and galactic clock. In setting on the responsibilities of the sensor, the lighting is either traded ON/OFF or decreased. The reducing advancement is by controlling the information voltage to the mechanical social affair or driver. The control can be either individual or in get-together.

D.A Presence Sensor for Smart Lighting Systems, Leo H. Botler, 2016.

Knowing whether a room is fused or not is essential for refreshing electrical criticalness reasonableness. For instance, if a given room is unfilled there is no requirement for the lights to be turned on. Ordinarily in little rooms, for instance, lift ways, a PIR sensor is used together with the lighting, at any rate as it nonattendances of precision, people dependably are left in uncertain quality after a few minutes. In the present work a contraption fit for including people a room is recognized and analyzed. It is what's more isolated and obvious game-plans [6].

E.Establishment of Vision Effect Diagram for Optimization of Smart LED Lighting, Qi Yao, Lei Yuan, YuBian., 2016.

In this paper, a structure appearing to be joined circadian effect and metopic vision impact recognized by cerotic, isotopic, and photonic photoreceptors, i.e., a fantasy impact chart, was set up using a chromaticity diagram subject to L, $\mathrm{M}$, and S cone cells for reference. The characteristics of the vision impact chart were bankrupt down, and diverse features clearly looked like those of the reference chromaticity plot. The connection between the vision impact and the chromaticity graphs was equivalently thought to ensure that the circadian and mesopic vision effects would be open in the perfect chromaticity broaden. The vision impact chart is a numerical contraption that can be used to find and foresee circadian and mesopic vision effects of light sources, similarly as to update light sources to induce these effects. Instigated light sources with both charming circadian and mesopic vision effects would not solely be significant when these two vision impacts are revived together yet would do well in future shrewd Light Transmitting Diode (LED) lighting with adaptable luminance in various outside applications moreover [7].

\section{PROPOSED SYSTEM}

In our proposed system we are going to monitor the street lights which are automatically turned on upon the surrounding weather condition.

\section{SYSTEM MODULES}

\section{A. Arduino Mega}

The Mega 2560 is a microcontroller board subject to the ATmega2560. It has 54 moved information/yield pins (of which 15 can be utilized as PWM yields), 16 direct information sources, 4 UARTs (gear dynamic ports), a 16 $\mathrm{MHz}$ productive stone oscillator, a USB affiliation, a power jack, an ICSP header, and a reset catch. It contains everything expected to help the microcontroller; on a fundamental dimension interface it to a PC with a USB alliance or power it with an AC-to-DC connector or battery to begin. The Mega 2560 board is phenomenal with most shields arranged for the Uno and the past sheets Duemilanove or Diecimila.

\section{B. LDR Sensor}

A Light Dependent Resistor (moreover called LDR, photoconductor, or photocell) is a contraption which has a constraint which moves as shown by the degree of light falling on its surface. A standard light ward resistor is appeared together with (on the right hand side) its circuit group picture. Assembled LDR's have unquestionable subtleties, notwithstanding the LDR's we move in the REUK Shop are very standard and have a constraint in full scale shadowiness of $1 \mathrm{Ohm}$, and an obstruction of a couple Ohm in radiant light (10-20kOhm @ 10 lux, 2-4kOhm @ 100 lux). Utilizations for Light Dependent Resistors. Light poor 
resistors are a central part in any electric circuit which is to be turned on and off thus as demonstrated by the fragment of consolidating light - for example, sun based controlled greenery fenced in an area lights, and night security lighting.

\section{C. $L C D$}

LCD (Liquid Crystal Display) screen is an electronic presentation module and locate a wide dimension of occupations. A $16 \times 2$ LCD show is principal module and is commonly utilized in different gadgets and circuits. These modules are supported more than seven sections and other multi area LEDs. The reasons being: LCDs are moderate; conceivably programmable; have no necessity of showing up and even custom characters (in no way like in seven sections), activities, etc. The ask for register stores the heading rules given to the LCD. An ask for is a course given to LCD to complete a predefined errand like appearing, clearing its screen, setting the cursor position, controlling presentation, and so on. The information register stores the information to be showed up on the LCD. The information is the ASCII estimation of the character to be showed up on the LCD. Snap to get settled with inside structure of a LCD.

\section{D.IoT Module}

The snare of things (IoT) is the game-plan of physical devices, vehicles, structures and diverse things embedded with equipment, programming, sensors, actuators, and framework organize that attract these articles to store up and exchange data. In 2013 the Global Standards Initiative on Internet of Things (IoT-GSI) portrayed the IoT as "the structure of the information society. The IoT connects with articles to be seen and controlled remotely transversely over existing structure system, making open sections for consistently direct blend of the physical world into PC based structures, and achieving improved profitability, accuracy and money related great position. Accurately when IoT is extended with sensors and actuators, the enhancement changes into an event of the more wide class of advanced physical structures, which also joins sorts of advancement, for instance, clever cross sections, splendid homes, insightful transportation and shrewd urban zones. Everything is astoundingly clear through its embedded enrolling structure yet can interoperate inside the present Internet establishment. Experts measure that the IoT will incorporate in every practical sense 50 billion things by 2020 .

\section{E. IR Sensor}

IR sensor have Emitter and locator The creator is just an IR LED (Light Emitting Diode) and the pioneer is fundamentally an IR photodiode which is faulty to IR light of foggy wavelength from that transmitted by the IR LED. Right when IR light falls on the photodiode, the affirmations and these yield voltages, change in degree to the hugeness of the IR light got.

\section{V.SYSTEM ARCHITECTURE}

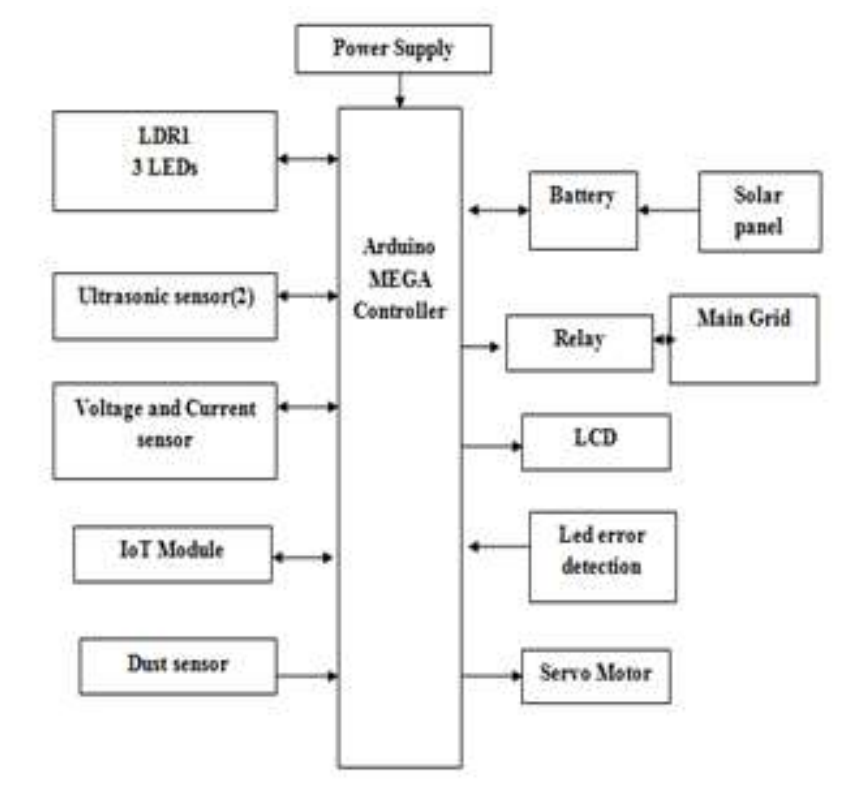

Fig. 1. System Architecture

\section{A. Advantages}

- It is close to nothing and increasingly clear to learn, grasp, program and examine.

- Compared to low dimension processing develop, C code made is dynamically strong and versatile, progressively adaptable between different stages.

- C compilers are available for all intents and purposes all introduced devices being utilized today and there is a tremendous pool of experienced $\mathrm{C}$ programming engineers.

- It is really capable.

- It supports access to I/O and gives effortlessness of the leading body of huge embedded exercises.

\section{FUTURE ENHANCEMENT \& RESULTS}

It is crucial to diminish the essentialness use and development the bundle level of this module to make it versatile. This will be the reason for mixing of the makers' future work, which besides joins playing out an irrefutably down to business and controlled botch examination, testing the contraption emanated light with change systems, for instance, PWM, testing with more people, and testing in multidoor conditions. The full scale structure should in like way join an IoT interface, which will require an extra module.

\section{CONCLUSION}

In this work, we present another framework for constraining issues in EUD for IoT dependent on semiotic arranging. We base on the insight of these frameworks and their obvious areas, a philosophy that keeps an eye out for the various parts of EUD in the IoT space, going from programming and device points of view to the setting of use. By applying this obliging in the IFTTT + Hue case, we 
depicted how our system can uncover a couple of issues that have not been investigated yet and that point at various issue definitions and, subsequently, make the open area for various issue philosophies. Doing everything considered with no other individual's data has the confirmation to build up our insight about EUD in intriguing ways. Our technique is central, at any rate we imagine dazzling future blend building up a positive structure to take a gander at such a muddled collecting correspondence situation, unquestionably a touch of its key viewpoints. Hence, our present response to the intrigue showed up on the title of this paper is that a wise light is so captured by parity of its coursed interface and the need to perceive a gander at it as a contraption submerged it as for a dynamically obvious typical plan of gadgets, applications and, explicitly, individuals. A HCC way to deal with oversee coordinates EUD for IoT dependably drives our idea concerning the probability of the correspondence between individuals, modelers and clients, through spread interfaces of various sorts.

\section{REFERENCES}

1. Wang, Yuan, and Partha Dasgupta. "Designing an adaptive lighting control system for smart buildings and homes." In 2015 IEEE 12th International Conference on Networking, Sensing and Control, pp. 450-455. IEEE, 2015.

2. Garg, Vijay Kumar. "Advance Lighting and Water Pumping System using Artificial Intelligence." In 2018 Fifth International Conference on Parallel, Distributed and Grid Computing (PDGC), pp. 1-7. IEEE, 2018.

3. Abinaya, B., S. Gurupriya, and M. Pooja. "IoT based smart and adaptive lighting in street lights." In 2017 2nd International Conference on Computing and Communications Technologies (ICCCT), pp. 195-198. IEEE, 2017.

4. Castro, Miguel, Antonio J. Jara, and Antonio FG Skarmeta. "Smart lighting solutions for smart cities." In 2013 27th International Conference on Advanced Information Networking and Applications Workshops, pp. 1374-1379. IEEE, 2013.

5. Martirano, Luigi. "A smart lighting control to save energy." In Proceedings of the 6th IEEE International Conference on Intelligent Data Acquisition and Advanced Computing Systems, vol. 1, pp. 132-138. IEEE, 2011.

6. Leo H. Botler, Djamel H. Sadok. "A presence sensor for smart lighting systems", IECON 2016 - 42nd Annual Conference of the IEEE Industrial Electronics Society, 2016

7. Yao, Qi, Lei Yuan, and Yu Bian. "Establishment of vision effect diagram for optimization of smart LED lighting." IEEE Photonics Journal 8, no. 4 (2016): 1-8.

8. Matta, Sherif, and Syed Masud Mahmud. "An intelligent light control system for power saving." In IECON 2010-36th Annual Conference on IEEE Industrial Electronics Society, pp. 3316-3321. IEEE, 2010.

9. Singhvi, Vipul, Andreas Krause, Carlos Guestrin, James H. Garrett Jr, and H. Scott Matthews. "Intelligent light control using sensor networks." In Proceedings of the 3rd international conference on Embedded networked sensor systems, pp. 218-229. ACM, 2005.

10. Ihm, Pyonchan, Abderrezek Nemri, and Moncef Krarti. "Estimation of lighting energy savings from daylighting." Building and Environment 44, no. 3 (2009): 509-514.

11. Lu, Jiakang, and Kamin Whitehouse. "SunCast: fine-grained prediction of natural sunlight levels for improved daylight harvesting." In Proceedings of the 11th international conference on Information Processing in
Sensor Networks, pp. 245-256. ACM, 2012.

12. Bhairi, Maheshkumar Narsayya, Shubhangi Shital Kangle, Manohar Suresh Edake, Bhaskar Shivraj Madgundi, and V. B. Bhosale. "Design and implementation of smart solar LED street light." In 2017 International Conference on Trends in Electronics and Informatics (ICEI), pp. 509-512. IEEE, 2017.

13. Bhanumathi, S., M. Vineeth, and N. Rohit. "Crop Yield Prediction and Efficient use of Fertilizers." In 2019 International Conference on Communication and Signal Processing (ICCSP), pp. 0769-0773. IEEE, 2019.

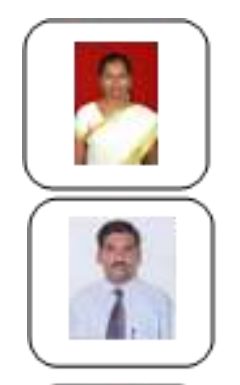

\section{AUTHORS PROFILE}

Nirmalrani $\mathbf{V}$ is an Assistant Professor in School of Computing, Sathyabama Institute of Science and Technology.

Saravanan $\mathbf{P}$ is an Assistant Professor in School of Computing, Sathyabama Institute of Science and Technology.

Sowmiya $\mathbf{G}$ is currently pursuing Bachelor of Technology in Information Technology. Her research interest is Internet of Things.

Peruma C is currently pursuing Bachelor of Technology in Information Technology. Her research interest is Internet of Things. 